\title{
ROLE OF VITAMIN D IN THE MANAGEMENT OF CHRONIC OBSTRUCTIVE PULMONARY DISEASE: AN OPTION OR NECESSITY
}

Amit Kumar Verma1, Sanjay Singh², Puneet Gupta ${ }^{3}$, Ashok Kumar Tripathi ${ }^{4}$

\section{HOW TO CITE THIS ARTICLE:}

Amit Kumar Verma, Sanjay Singh, Puneet Gupta, Ashok Kumar Tripathi. "Role of Vitamin D in the Management of Chronic Obstructive Pulmonary Disease: An Option or Necessity". Journal of Evolution of Medical and Dental Sciences 2015; Vol. 4, Issue 63, August 06; Page: 11063-11068, DOI: 10.14260/jemds/2015/1593

\begin{abstract}
COPD is one of few diseases where mortality and morbidity is on rise. It is a complex disease characterized by the airway and systemic inflammation, chronic airflow limitation, progressive tissue destruction etc. Inflammatory response in COPD is characterized by the increase in number of macrophages, neutrophils \& lymphocytes in airways and lung parenchyma. Airway inflammation further results in systemic inflammation and other COPD related manifestations. There is strong relationship between 25- hydroxyl Vitamin $\mathrm{D}$ and pulmonary functions as assesses by forced expiratory volume in one second $\left(\mathrm{FEV}_{1}\right)$ and forced vital capacity (FVC). The patients of COPD are also known to have low serum levels of Vit D3 either as a cause or consequence of disease. But vitamin D supplementation in the patients of COPD is still in stage of controversy as some reports have shown beneficial and others reported no significant benefit.
\end{abstract}

KEYWORDS: COPD, Oxidative stress, Vitamin- D, Inflammation, FEV1, Osteoporosis.

INTRODUCTION: COPD is one of few diseases, where morbidity and mortality is on rise. Worldwide, COPD is a health problem with severe economical and social effects. It results in approximately $5 \%$ of total deaths worldwide, is the fifth leading cause of death in the world, and is expected to become third by year $2030 .{ }^{1}$ At the personal level, COPD constitutes a major cause of patient disability and of low quality of life for patients and their caregivers. ${ }^{(2,3)}$

By definition COPD is a chronic and progressive reduction in airflow, secondary to an abnormal inflammatory response of the lungs to the inhalation of noxious particles or toxic gases. This inflammation produces alterations of varying severity in the bronchi (Chronic bronchitis), bronchioles (Obstructive bronchiolitis), lung parenchyma (Emphysema), or any combination of the above. 4,5

The inflammation and airflow reduction main components in pathophysiology of COPD The inflammation in COPD does not, significantly respond to steroids. The reduction in airflow in COPD has a significant irreversible component, secondary to structural changes in the airways, ${ }^{6,7}$ such as peribronchiolar fibrosis and increased collapsibility, resulting from the destruction of the lung tissue. These changes are triggered by a complex mechanism that initiates well before the first clinical and functional manifestations. ${ }^{8}$ Therefore, a better understanding of the mechanisms involved in the apparently complex etiopathogenesis of COPD will allow not only an earlier diagnosis but also the development of therapeutic agents that can favorably alter the course of the disease before the development of permanent structural changes.

\section{The factors Responsible for the Alterations Observed in COPD:}

1. Oxidative stress.

2. Inflammation.

3. Protease-antiprotease imbalance.

4. Apoptosis. ${ }^{8}$ 
Airway inflammation and oxidative stress have been implicated in the pathogenesis of COPD. The inflammation in COPD shows high numbers of neutrophils, macrophages and $\mathrm{T}$ lymphocytes, especially of the CD8 positive T cell subset in broncho-alveolar lavage fluid.8, There is increased levels of circulating cytokines, including $C$ reactive protein (CRP), tumor necrosis factor$\alpha$ (TNF- $\alpha$ ), interleukin-8 (IL-8), interleukin-6 (IL-6), interleukin-1 $\beta$ (IL-1 $\beta$ ), and adipokinase. IL-8 is a strong chemo-attractant of neutrophils; it also initiates degranulation, production of reactive oxygen species (ROS) and increased expression of cell adhesion molecule CD1b/CD18 (MAC-1).10 Cell adhesion molecules are major factors in recruitment, cumulating and site specific activation of inflammatory cells in the airways. ${ }^{11}$ TNF- $\alpha$ is an important inflammatory mediator produced by several kinds of cells, playing a role in activating the neutrophils and stimulating the release of IL-8. Its over-expression can lead to the chronicity of inflammatory response and the aggravation of lung injury.

The production of reactive species ROS, RNS is an integral part of metabolism and is present under normal conditions, i.e. in the physiological processes involved in the energy production, regulation of cell growth, phagocytosis, intracellular signaling and synthesis of hormones and enzymes. 12

In order to offset this production and its potential negative effects, the body has an antioxidant system. In situations in which there is an imbalance between the pro-oxidant system and the antioxidant system, oxidative stress occurs. ${ }^{12}$ Oxidative stress plays an important role in the pathogenesis of COPD through direct injurious effects in lungs but also activates molecular mechanisms that initiate lung inflammation. ${ }^{13}$ Several studies show relationships between oxidative stress markers and the degree of airflow limitation in COPD.

The concept of link between COPD, serum Vitamin D status is just around on decade old when in year 2005 Black et.al. during the data analysis of (Third national health and nutrition survey) NHANES III study, found that after the adjustment of potential co-founders including intake of supplements and outdoor activities, a strong relationship was between serum levels of vitamin D and lung function (FEV1 and FVC). Although no association between 25-OH Vitamin D concentrations and FEV1/ FVC ratio was found. ${ }^{7}$

There are several factors that could account for the Vitamin D deficiency in COPD patients: poor diet, a reduces capacity of ageing skin for Vitamin D synthesis, reduced outdoor activity and therefor sun exposure, an increased catabolism by glucocorticoids, impaired activation because of renal dysfunction and lower storage capacity in muscles and fat due to wasting. ${ }^{14}$

In COPD, lung destruction is mediated partly through inflammation, oxidative stress, and increased release of proteases, and many of these processes are modulated by vitamin D. ${ }^{15}$ Cellular studies reveals that vitamin D modulates the activity of various defence and immune cells including monocytes, macrophages, lymphocytes both B and T types, or epithelial cells. ${ }^{16}$

Epithelial cells and macrophages express Vitamin D receptor(VDR) at high levels and possess the enzymatic machinery to produce 1,25-dihydroxyvitamin D3 locally in the lung (CYP27B1). ${ }^{17,18}$ Macrophages in particular respond to 1,25-dihydroxyvitamin D3 and thereby prevent excessive expression of inflammatory cytokines and chemokines.

On the top of above all no direct proof exists that vitamin D deficiency or impaired vitamin D signaling is causally involved in the pathogenesis of COPD. Likewise, whether activation of the vitamin D pathway, by supplementation or other strategies, alters the disease course remains to be explored. 
In human beings up to now, only one randomized controlled intervention study has investigated the effect of vitamin D supplementation on exacerbation risk. ${ }^{19}$ In this study done by Cavailles et al at University of Leuven, Belgium, 182 patients with moderate to very severe COPD were randomly assigned to receive either monthly doses of $100000 \mathrm{IU}$ cholecalciferol or placebo for 1 year.

The results were mean serum 25-(OH)D levels increased significantly in the vitamin D group compared with the placebo group(mean between-group difference, 30mg/mL [95\% CI, 27 to $33 \mathrm{mg} / \mathrm{mL}$; $\mathrm{P}<0.001)$. The median time to first exacerbation did not significantly differ between the groups (hazard ratio, 1.1 [CI, 0.82 to 1.56]; P 0.41), nor did exacerbation rates, FEV1, hospitalization, quality of life, and death. However, a post hoc analysis in 30 participants with severe vitamin D deficiency (serum 25-[OH] D levels $10 \mathrm{mg} / \mathrm{mL}$ ) at baseline showed a significant reduction in exacerbations in the vit. D group.(rate ratio, 0.57 [CI, 0.33 to0.98]; $\mathrm{P}=0.042$ ). The study found a relative improvement of monocyte phagocytosis capacity in patients who were supplemented, especially in the deficient subgroup. Because of low sample size, limited follow up and the specific setting of severe disease under maximum treatment, the trial was not able to refute the potential of long-term vitamin $\mathrm{D}$ intervention for the reduction of exacerbations or lung function decline in patient cohort with mild disease.

To add on COPD has many comorbidities including coronary heart disease, heart failure and lung cancer, for them smoking is a common risk factor. Other comorbidities such as pulmonary artery disease and malnutrition are directly caused by COPD, whereas many like systemic venous thromboembolism, anxiety, depression, osteoporosis, obesity, metabolic syndrome, diabetes, sleep disturbance and anaemia, have no evident physiopathological relationship with COPD. The common ground between most of these extrapulmonary manifestations is chronic systemic inflammation. ${ }^{20}$

Out of these co morbidities lets discuss osteoporosis. Along with osteoporosis COPD also occurs in age groups of 40 or more. The respiratory complications of osteoporosis are even more marked in COPD because they exacerbate the physical inactivity and increase the risk of vertebral fractures, with total lung capacity becoming progressively reduced.

Epidemiological studies show that the prevalence of osteoporosis in COPD patients is increased. GRAAT-VERBBOM et al. ${ }^{21}$ Conducted a systematic review of 13 cross-sectional studies published between 1998 and 2008, including a total of 775 COPD patients. The analytical methods and definitions of osteoporosis varied between the studies. According to the World Health Organization definition and based on bone densitometry, the prevalence of osteoporosis ranged from $24 \%$ to $69 \%$. The COPD patients with osteoporosis had significantly lower FEV1 and BMI values than those without osteoporosis. There is a negative correlation between osteoporosis and FEV1 values. Some therapeutic trials include a placebo arm to take into account the natural course; the duration of these trials was nevertheless limited to a few years.

MINEO et al. ${ }^{22}$ evaluated the bone density of 40 patients before and 1 year after lung volume reduction surgery for emphysema. It suggested that this surgery improved bone density, despite oral steroid therapy, which was maintained after surgery in over half of the patients.

In the Evaluation of Obstructive Lung Disease and Osteoporosis (EOLO) study, the prevalence of vertebral fractures in an ambulatory COPD population was 41\%, and correlated with COPD severity. ${ }^{23}$ 


\section{REVIEW ARTICLE}

\section{At the Cellular Level, Bone Remodeling is regulated by three Effectors:}

1. The osteoblasts that form the bonematrix, which is subsequently calcified;

2. The osteoclasts that resorb bone; and

3. The osteocytes that coordinate bone remodeling.

The known shared risk factors for COPD and osteoporosis are Smoking and systemic inflammation affect RANK/RANKL binding, and vitamin D deficiency stimulates parathormone secretion and affects osteoclast maturation through its effect on RANK/RANKL. Recent data show that inflammatory cytokines can also target Wnt. Vitamin D can also improve the expression of the osteoprotegerin/RANKL complex, reducing osteoclast formation.

Oral steroid therapy affects bone homeostasis through increased expression of RANKL, decreased osteoprotegerin and suppression of osteoclast apoptosis. The hypogonadism present in COPD also plays a part in the development of osteoporosis: the drop in oestrogen levels inhibits the action of the osteoprotegerin/RANKL complex.

Although an association between smoking and osteoporosis has been established, the studies do not report lung function values. ${ }^{24}$ the lower levels of physical activity commonly found in COPD patients have a negative effect on bone metabolism, a phenomenon that has been clearly demonstrated in post-menopausal females. A negative impact of vitamin D deficiency on bone homeostasis has been suggested: its frequency increases with COPD stage, reaching $77 \%$ for the very severe stage according to JANSSENS et al. ${ }^{25}$ More generally, malnutrition has a negative impact and is another common finding in COPD.

Pro-inflammatory cytokines, such as IL-1b, IL-6 and TNF-a, may promote bone resorption. The harmful effect of oral steroid therapy on bone metabolism has long been established, and recent studies of the underlying mechanism have highlighted the impact of oral steroid therapy on both bone resorption and impaired bone formation.

Calcium and vitamin D supplementation have proven effective in reducing fracture risk, the effect being dose-dependent for vitamin $\mathrm{D}$ and only when combined with calcium. ${ }^{26}$ Several recent studies on vitamin D have found it to improve muscle function and postural stability, and to have immunomodulatory effects. A daily dose of 800 IU of vitamin D combined with calcium supplementation is recommended for patients with a T-score of,-1 and three minor risk factors (BMI, $21 \mathrm{~kg} / \mathrm{m}^{2}$, active smoking, chronic alcohol abuse, age. 65 years, hip fracture, rib fracture, menopause, sedentary lifestyle andFEV1, 50\% predicted) or one major risk factor (Systemic corticosteroid therapy for $>3$ months per year, previous vertebral compression fracture). Bisphosphonate therapy has not been specifically evaluated for osteoporosis in COPD patients, although research by SMITH et al. ${ }^{27}$ suggests it has a beneficial effect on the T-scores of patients with airways disease.

The usefulness in COPD of new treatments such as teriparatide remains to be defined, whereas its role in the treatment of glucocorticoid-induced osteoporosisis now well established.28

From these evidences it is clear that serum Vitamin D levels in COPD patient are generally low either due to its pathogenesis or secondary outcome of disease. In Indian clinical scenario $>90 \%$ patients are found to have serum vitamin D 3 levels $<30 \mathrm{pg} / \mathrm{ml}$, although it does not correlates with severity of disease.

In the opinion of authors in the best interest of patients attempt should be made to keep serum vitamin D 3 to sufficient levels either with diet or drug. It may not improve lung functions in COPD patients but it will certainly manage osteoporosis/ osteopenia and reduce the risk of bone fractures in them. 


\section{BIBLIOGRAPHY:}

1. World Health organization (WHO) The global burden of Disease: 2004 update. Available at: http://www.who.int/healthinfo/global_burden_disease/GBD_reprt_2004update_full.pdf. Accessed October 2014.

2. Pinto RA, Holanda MA, Medeiros MM, Mota RM, Pereira ED. Assessment of the burden of caregiving for patients with chronic obstructive pulmonary disease. Respir Med. 2007; 101: 2402-8.

3. Nunes DM, Mota RM, Neto OL, Pereira ED, de Bruin VM, Bruin PF. Impaired sleep reduces quality of life in chronic obstructive pulmonary disease. Lung. 2009; 187: 159-63.

4. Sethi s, Wrons $\mathrm{C}$, Eschberger $\mathrm{K}$, et al. Inflammatory profile of new bacterial strain exacerbations of COPD. Am J Respir Crit Care Med. 2008; 177:491-97.

5. Pinto-Plata VM, Livnat G, Girish M, et al. Systemic cytokines, clinical and physiological changes in patients hospitalized for exacerbation of COPD. Chest. 2007; 131:37-43.

6. Rohde G, G Evaert P, Holtappels G et al. Soluble interleukin 5 alpha receptor is increased in acute exacerbation of COPD. Int Arch All Immunol. 2004; 135:54-61.

7. Black PN, Scragg R. Relationship between serum 25 hydroxy vitamin-d and pulmonary function in the third national health and nutritional examination survey. Chest 2005; 128:3792-8.

8. Majo J, Ghezzo H, Cosio MG. Lymphocyte population and apoptosis in the lungs of smokers and their relation toemphysema. Eur Respir J 2001; 17:946-53.

9. Jeffrey PK. Lymphocytes, chronic bronchitis and chronic obstructive pulmonary disease. Novartis Found Symp2001; 234:149-61.

10. Noguera A, Batle S, Miralles C, Lglesias J, Busquets X. Enhanced neutrophil response in chronic obstructive pulmonary disease. Thorax 2001; 56:432-7.

11. Roche WR, Montefort S, Baker J, Holgate ST. Cell adhesion molecules and the bronchial epithelium. Am Rev Respir Dis1993; 148:S79-82.

12. Rajendra sozhan S, Yang SR, Edirisinghe I, Yao H, Adenuga D, Rahman I. Deacetylase and NFkappa B in redox regulation of cigarette smoke-induced lung inflammation: epigenetics in pathogenesis of COPD. Antioxid Redox Signal. 2008; 10:799-811.

13. Rahman I, Macnee W. Role of transcription factors in inflammatory lung diseases. Thorax 1998; 53:601-612.

14. HolickMF: VitaminD deficiency. N Engl J Med2007, 357: 266-281.

15. Baeke F, Takiishi T, Korf H, Gysemans C, Mathieu C. Vitamin D:modulator of the immune system. Curr Opin Pharmacol 2010; 10: 482-96.

16. Sethi S, Donald AM, Marcus P, Owen CA, Yawn B, Rennard S: Inflammation in COPD: Implication for management. The am J of Med 2012; 125, 1162-70.

17. Hansdottir S, Monick MM, Hinde SL, Lovan N, Look DC, Hunninghake GW. Respiratory epithelial cells convert inactive vitamin $\mathrm{D}$ to its active form: potential effects on host defense. JImmunol 2008; 181: 7090-99.

18. Overbergh L, Decallonne B, Valckx D, et al. Identification and immune regulation of 25hydroxyvitamin D-1-alpha-hydroxylase inmurine macrophages. ClinExpImmunol 2000; 120: 139-46.

19. LehouckA, Mathieu C, CarremansC, BaekeC, VerhaegenJ, EldereJV, Decallonne B. Bouillon R, Decrame $M$ Janssens W. High Doses of Vitamin $D$ to Reduce Exacerbations in ChronicObstructive Pulmonary Disease. Ann Intern Med. 2012; 156:105-114. 
20. Cavailles A, Robin GB, Dixnies A, Goupi F, Gobert CG et.al. Co morbidities in OPD. Eur Respir Rev 2013; 22: 454-475 | DOI: 10.1183/09059180.00008612.

21. Graat-Verboom L, Wouters EFM, Smeenk FW, et al. Current status of research on osteoporosisin COPD: asystematic review. Eur Respir J 2009; 34: 209-218.

22. Mineo TC, Ambrogi V, Mineo D, et al. Bone mineral density improvement after lung volume reduction surgery for severe emphysema. Chest 2005; 127: 1960-1966.

23. Maggi S, Siviero P, Gonnelli S, et al. EOLO Study Group. Osteoporosis risk in patients with chronic obstructive pulmonary disease: the EOLO study. J ClinDensitom2009; 12: 345-352.

24. Ionescu AA, Schoon E. Osteoporosis in chronic obstructive pulmonary disease. Eur Respir J 2003; 22: Suppl. 46,64s-75s.

25. Janssens W, Bouillon R, Claes B, et al. Vitamin D deficiency is highly prevalent in COPD and correlates withvariants in the vitamin D-binding gene. Thorax 2010; 65: 215-220.

26. Avenell A, Gillespie WJ, Gillespie LD, et al. Vitamin D and vitamin D analogues for preventing fractures associated with involutional and post-menopausal osteoporosis. Cochrane Database Syst Rev 2009; 2: CD000227.

27. Smith BJ, Laslett LL, Pile KD, et al. Randomized controlled trial of alendronate in airways disease and low bonemineral density. Chron Respir Dis 2004; 1: 131-137.

28. Grossman JM, Gordon R, Ranganath VK, et al. American College of Rheumatology 2010 recommendations forthe prevention and treatment of glucocorticoid-induced osteoporosis. Arthritis Care Res (Hoboken) 2010; 62:1515-1526.

\section{AUTHORS:}

1. Amit Kumar Verma

2. Sanjay Singh

3. Puneet Gupta

4. Ashok Kumar Tripathi

\section{PARTICULARS OF CONTRIBUTORS:}

1. Assistant Professor, Department of TB \& Chest, University College of Medical Sciences, Dilshad Garden, Delhi.

2. Assistant Professor, Department of TB \& Respiratory Diseases, Career Institute of medical Sciences, Hardoi Bypass Road, Ghailla, Lucknow.

3. Consultant Pulmologist, Kailash Hospital, Noida, U. P.

FINANCIAL OR OTHER COMPETING INTERESTS: None
4. Professor, Department of Bio Chemistry, University College of Medical Sciences, Dilshad Garden, Delhi.

\section{NAME ADDRESS EMAIL ID OF THE CORRESPONDING AUTHOR:}

Dr. Amit Kumar Verma, Room. No-1605, Ward-16, GTB Hospital Dilshad Garden, Delhi-110095.

E-mail: dramitkverma@hotmail.com

Date of Submission: 11/07/2015. Date of Peer Review: 13/07/2015. Date of Acceptance: 29/07/2015. Date of Publishing: 06/08/2015. 